\title{
Zinc and Hepatocyte Nuclear Factor-4 $\alpha$ in Alcohol-Induced Intestinal Barrier Dysfunction
}

\author{
Zhanxiang Zhou* and Wei Zhong
}

Department of Nutrition, University of North Carolina at Greensboro, Greensboro, NC, USA

\begin{abstract}
Zinc is an essential micronutrient and plays an important role in maintaining intestinal epithelial integrity. Increasing evidence support that zinc homeostasis has significant impact on the intestinal barrier via regulation of epithelial tight junction proteins. Intestinal barrier plays a critical role in the prevention of endotoxin penetration from the intestinal lumen to the blood. Disruption of the intestinal barrier leads to elevation of blood endotoxin level, namely endotoxemia. Endotoxemia may lead to proinflammatory cytokine production and inflammation, thereby being an etiological factor in the pathogenesis of alcoholic liver disease (ALD). Recent studies demonstrated that oxidative stress and zinc deficiency correlate well with alcohol-induced gut leakiness. Alcohol exposure induces oxidative stress which, in turn, releases zinc from proteins. Hepatocyte nuclear factor- $4 \alpha(\mathrm{HNF}-4 \alpha)$ is a zinc finger transcription factor which abundantly distributes in the intestine, particularly the distal intestine. Inactivation of HNF- $4 \alpha$ correlates well with alcohol-induced downregulation of tight junction proteins. This review discusses mechanisms involved in alcohol-induced intestinal epithelial barrier disruption with emphasis on the relationship among oxidative stress, zinc deficiency, and HNF- $4 \alpha$ inactivation.
\end{abstract}

Keywords: Zinc deficiency, HNF-4 $\alpha$ get permeability, alcoholic liver disease.

\section{INTRODUCTION}

Zinc is the second most abundant trace element in the body next to iron. Zinc critically participates in a variety of cellular functions such as metabolism, cell signaling and gene expression. While zinc ion alone may have antioxidant property by its antagonism to transitional metals in free radical formation [1], zinc impacts cellular functions mainly by binding to large number of proteins including enzymes, transcription factors and structural proteins. Zinc commonly binds to cysteine and/or histidine residues to form zinc/sulfur complex, a catalytic or a structural site of proteins [2,3]. However, the zinc/sulfur binding sites are redox sensitive, and zinc release from its binding sites will lead to inactivation of proteins. Reactive oxygen species (ROS) have been shown to release zinc from the zinc finger, leading to loss of DNA binding activity of zinc finger transcription factors $[4,5]$. Under chronic disease condition, sustained oxidative stress may not only inactivate zinc proteins, but also reduce the total zinc concentrations in organs, i.e. zinc deficiency.

Recent studies provide strong evidence that zinc critically regulates epithelial tight junction proteins and the epithelial barrier in a variety of epithelial cells [6-13]. Epithelial barrier is known to play an important role in macromolecule transport and organ function. In particular, the intestinal barrier prevents endotoxin penetration from the intestinal lumen to the blood, thereby serving as a systemic guarder. Disruption of the intestinal barrier and development of endotoxemia have been reported in disease conditions such as enterotitis,

*Address correspondence to this author at the 500 Laureate Way, Suite 4226, Kannapolis, NC 28081; Tel: 704-250-5800; Fax: 704-250-5809;

E-mail: z_zhou@uncg.edu and alcoholic and non-alcoholic steatohepatitis [14-17]. Zinc plays a critical role in regulation of the intestinal barrier integrity and epithelial repair [18]. This review discusses mechanisms involved in alcohol-induced intestinal epithelial barrier dysfunction with emphasis on the relationship among oxidative stress, zinc deficiency, and HNF- $4 \alpha$ inactivation.

\section{ALCOHOL-INDUCED GUT LEAKINESS}

ALD is a common health problem, but the mechanisms of how alcohol induces liver damage have not been fully understood. Increasing evidence support that the extrahepatic factors such as endotoxin may critically contribute to alcohol-induced liver damage [19]. Endotoxins are derived from the cell wall of Gram-negative bacteria that inhabit the lumen of the terminal intestine. Endotoxins normally penetrate the gut epithelium only in trace amounts; however, the penetration can be accelerated under pathophysiological conditions, leading to the elevation of blood endotoxin level, namely endotoxemia. Endotoxemia has been well documented in patients with ALD [20], and the blood endotoxin levels correlate well with TNF- $\alpha$ levels and the severity of ALD [21-23]. Animal studies demonstrated that abrogating endotoxin signal cascade in the liver by inhibition of Gramnegative bacteria with probiotics and antibiotics [24, 25] or neutralization of circulating endotoxin [26], led to attenuation of alcohol-induced cytokine production and liver damage. These findings suggest that endotoxemia is an important determinant in the pathogenesis of ALD.

Intestinal barrier dysfunction has been repeatedly reported in alcohol-induced endotoxemia and liver damage. Alcoholic patients showed increased gut permeability to a variety of permeability markers, such as polyethyleneglycol, 
lactulose/mannitol, or ${ }^{51}$ CrEDTA [27-30]. In animal studies, gut permeability to macromolecules such as horse radish peroxidase was also increased in association with alcoholinduced endotoxemia and liver damage [31-35]. Orally administrated lipopolysaccharide can be detected in the plasma of alcohol-intoxicated mice but not in the control mice [36]; this provides direct evidence that disruption of the intestinal barrier is responsible for alcohol-induced endotoxemia. A recent study showed that the gut leakiness in mice after chronic alcohol exposure occurs in the ileum, but not in the duodenum or jejunum, suggesting that the distal intestine may play an important role in the development of alcoholic endotoxemia [12]. Previous studies showed that intestinal histopathological alterations such as loss of epithelial cells at the top of the intestinal villi were associated with acute alcohol intoxication-induced intestinal barrier disruption [36]. However, chronic alcohol exposure reduced the distribution of tight junction proteins, but did not significantly affect the intestinal histopathology [12]. Thus, disassembly of tight junction proteins is likely a causal factor in the pathogenesis of chronic alcohol exposure-induced intestinal barrier dysfunction.

\section{ALCOHOL-INDUCED DISASSEMBLY OF INTESTI- NAL TIGHT JUNCTION PROTEINS}

The intestinal barrier is composed of the mucosal epithelial cells and the paracellular apical junction complex, in particular the most apical tight junctions [37]. Tight junctions seal the intercellular space to prevent the diffusion of macromolecules such as endotoxin from the intestinal lumen into the blood. Tight junctions are composed of several transmembrane proteins such as occludin and claudins and intracellular molecules such as zonula occludens 1 (ZO-1) [38]. The interactions of the cell surface molecules ensure intercellular adhesion and regulate paracellular permeability. Disruption of proteins at the tight junctions will result in dysfunction of the intestinal barrier to allow the diffusion of endotoxin from the intestinal lumen into the blood. Recent studies demonstrated that disruption of tight junction proteins appears to be a common mechanism involved in the pathogenesis of endotoxemia in a number of gastrointestinal diseases such as ALD, inflammatory bowel disease and celiac disease [30, 39, 40].

Alcohol exposure has been reported to disassemble tight junction proteins of the intestinal epithelium in association with epithelial barrier dysfunction. Alcohol exposure in Caco-2 cells produced a progressive disruption of tight junction protein ZO-1 with separation of ZO-1from the cellular junctions, leading to formation of large gaps between adjacent cells [8]. Alcohol exposure also produced a disassembly and displacement of perijunctional actin and myosin filaments from the perijunctional area [8]. Alcohol consumption in human and animals also increased the colon acetaldehyde level [41, 42]. Acetaldehyde exposure in Caco-2 cells disrupted the epithelial barrier in association with disassembly of tight junction proteins [43]. Inhibition of alcohol metabolism by 4-methylpyrazole attenuated alcohol-induced epithelial barrier disruption in Caco-2 cells, suggesting that acetaldehyde is more harmful than ethanol (our unpublished data). Colon biopsies from patients with ALD exhibited a reduction of the ZO-1 protein levels in comparison with normal sub- jects [44]. Our recent study [12] demonstrated a reduced distribution of tight junction proteins, occludin (Fig. 1A) and $\mathrm{ZO}-1$, in the distal intestine in association with increased gut permeability (Fig. 1B) and plasma endotoxin level (Fig. 1C) in mice chronically fed alcohol. Thus, disassembly of tight junction proteins is likely an important mechanism underlying alcohol-induced intestinal barrier dysfunction.

\section{OXIDATIVE STRESS AND ZINC DEFICIENCY IN ALCOHOL-IMPAIRED INTESTINAL EPITHELIAL BARRIER}

Oxidative stress has been a major research focus in understanding the mechanism of how alcohol exposure interferes with the epithelial barrier. Alcohol exposure has been shown to induce oxidative stress in association with increased activities of cytochrome P450 2E1, xanthine oxidase, and inducible nitric oxide synthase $[35,45,46]$ in the intestine. Oral administration of antioxidants attenuated alcohol-induced gut leakiness [32, 34, 47, 48]. ROS and nitric oxide production in Caco-2 cells were detected after alcohol treatment, and oxidation and nitration of tubulin was found in association with epithelial barrier disruption [49]. Further studies showed that hydrogen peroxide $\left(\mathrm{H}_{2} \mathrm{O}_{2}\right)$ and peroxynitrite $\left(\mathrm{ONOO}^{-}\right)$are responsible for oxidative modification of tight junction proteins [50-52]. NF- $\mathrm{KB}$ activation plays a critical role in oxidant-induced epithelial barrier dysfunction [53]. Treatment with antioxidants such as L-cysteine, superoxide dismutase and rebamipide, iNOS inhibitors, and growth factors such as epididymal growth factor or TGF- $\beta$ inhibited alcohol-induced ROS accumulation, tubulin nitration and epithelial barrier dysfunction [53-55]. These findings suggest that oxidative stress is a causal factor in alcohol-induced epithelial barrier disruption.

Increasing evidence suggest that oxidative stress disturbs cellular zinc homeostasis, leading to zinc deficiency [56]. Zinc deficiency has been well documented in patients with ALD, and the zinc levels in the serum and liver of patients with ALD were significantly lower than that of normal controls [57]. Our recent study demonstrated that zinc deficiency was associated with alcohol-induced oxidative stress and intestinal barrier disruption [12]. Chronic alcohol exposure in mice caused significant accumulation of ROS in the ileum, but not in the duodenum or jejunum (Fig. 2A), which correlated well with the measurements of gut permeability. Interestingly, the zinc levels in the ileum, but not in the duodenum or jejunum, were decreased by chronic alcohol exposure (Fig. 2B). Based on the fact that the mice were fed a zinc adequate diet in this study [12], the negative correlation between ROS and intestinal zinc level indicates that oxidative stress is likely the cause of alcohol-induced zinc deficiency in the intestine.

\section{IMPACT OF ZINC DEFICIENCY ON THE INTESTI- NAL EPITHELIAL BARRIER}

Prolonged zinc deficiency has been shown to induce morphological astrophy of intestinal mucosa [58]. Recent studies demonstrated a direct link between zinc deficiency and intestinal epithelial barrier dysfunction. Caco-2 cells cultured in zinc deficient medium exhibited a decrease in epithelial barrier function as indicated by lower values of trans-epithelial electronic resistance [8]. Zinc deficiency 

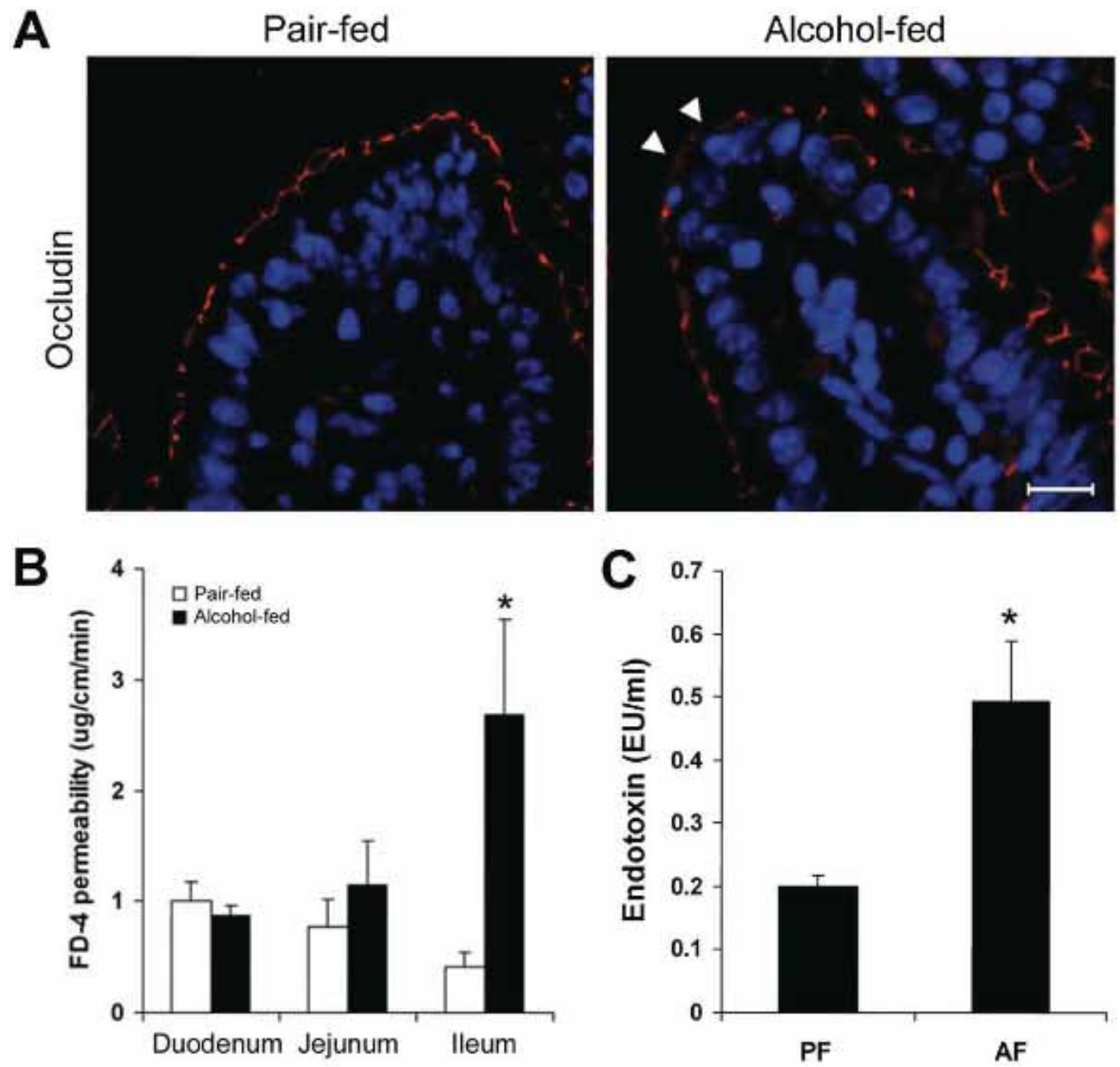

Fig. (1). Intestinal barrier dysfunction in mice chronically fed alcohol for 4 weeks. A. Immunofluorescence microscopy of the ileal tight junction protein, occludin. Arrowheads indicate the disappearance of occludin. Scale bar: $30 \mu \mathrm{m}$. B. Intestinal permeability. The penetration of intra-lumen FITC-Dextran ( $\mathrm{mw}$ 4,000) to the incubation buffer was determined after incubation of the intestinal sac of duodenum, jejunum and ileum for $20 \mathrm{~min}$. C. Plasma endotoxin. Endotoxin levels were measured by the limulus ameobocyte lystate method. Results are means \pm SD $(n=6-8)$. * Significantly different $(p<0.05, t$-test) from the pair-fed mice. PF: pair-fed, AF: alcohol-fed.
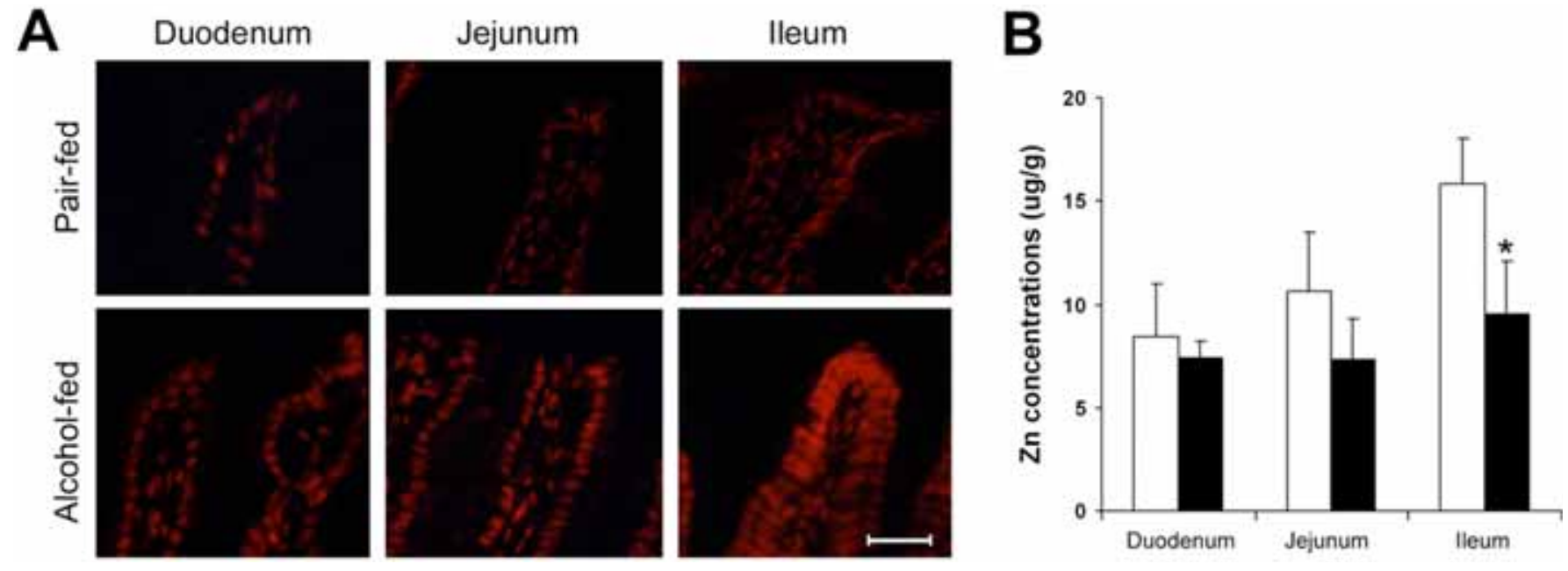

Fig. (2). Intestinal ROS accumulation and zinc concentrations in mice chronically fed alcohol for 4 weeks. A. Fluorescence microscopy of ROS. Cryostat intestinal sections were incubated with dihydroethidium at $5 \mu \mathrm{M}$ and red fluorescence was formed in the nuclei upon oxidation by ROS. Scale bar: $40 \mu \mathrm{m}$. B. Intestinal zinc concentrations. Total zinc was measured by atom absorption spectroscopy. Results are means \pm $\mathrm{SD}(n=6-8)$. * Significantly different $(p<0.05, t$-test) from the pair-fed mice. ROS: reactive oxygen species. 
caused delocalization of junction proteins including $\mathrm{ZO}-1$, occludin, $\beta$-catenin and E-cadherin, and disorganization of cytoskeleton proteins including $\mathrm{F}$-actin and $\beta$-tubulin. The protein levels of $\mathrm{ZO}-1$, occludin and $\beta$-tubulin were also reduced by zinc deficiency. In addition, zinc deficiency caused a dephosphorylation of occludin and hyperphosphorylation of $\beta$-catenin and ZO-1 [8]. Zinc deficiency also accelerated neutrophil migration through the Caco- 2 monolayer, and stimulated secretion of IL-8, epithelial neutrophil activating peptide-78, and growth-related oncogen-alpha [8]. These results suggest that zinc deficiency has deleterious effects on the epithelial barrier through impairing epithelial integrity and inducing inflammatory cell infiltration.

A Caco-2 alcohol exposure model was used to define the link among oxidative stress and zinc deprivation in alcoholinduced disruption of epithelial barrier [12]. Alcohol exposure increased cellular ROS and free zinc level in association with epithelial barrier disruption in Caco-2 cells (Fig. 3A). Supplementation with N-acetyl-cysteine (NAC) reduced cellular ROS and free zinc level in association with attenuating alcohol-induced epithelial barrier disruption (Fig. 3B).
These data suggest that zinc release from zinc proteins could be a downstream molecular event of oxidative stress in alcohol-induced epithelial barrier disruption. To define the link between zinc and epithelial junction proteins, Caco- 2 cells were cultured under zinc deficient condition which was achieved by adding a zinc chelator, N,N,N',N'-tetrakis(2pyridylmethyl)ethylenediamine (TPEN), in the media [12]. TPEN treatment impaired epithelial barrier function in a dose-dependent manner. Measurements of tight junction proteins demonstrated that zinc deficiency reduced the intercellular distribution (Fig. 4A) and protein levels (Fig. 4B) of occludin and other tight junction proteins including claudin1 and ZO-1. To eliminate the possible involvement of oxidative stress in the action of zinc deficiency, NAC was used to inhibition of oxidative stress. While inhibition of oxidative stress attenuated alcohol-induced cellular zinc release and epithelial barrier dysfunction, NAC treatment did not improve zinc deficiency-induced epithelial barrier dysfunction. These results indicate that the deleterious effects of zinc deficiency on the epithelial barrier integrity and function could be independent of oxidative stress. However, oxidative stress is

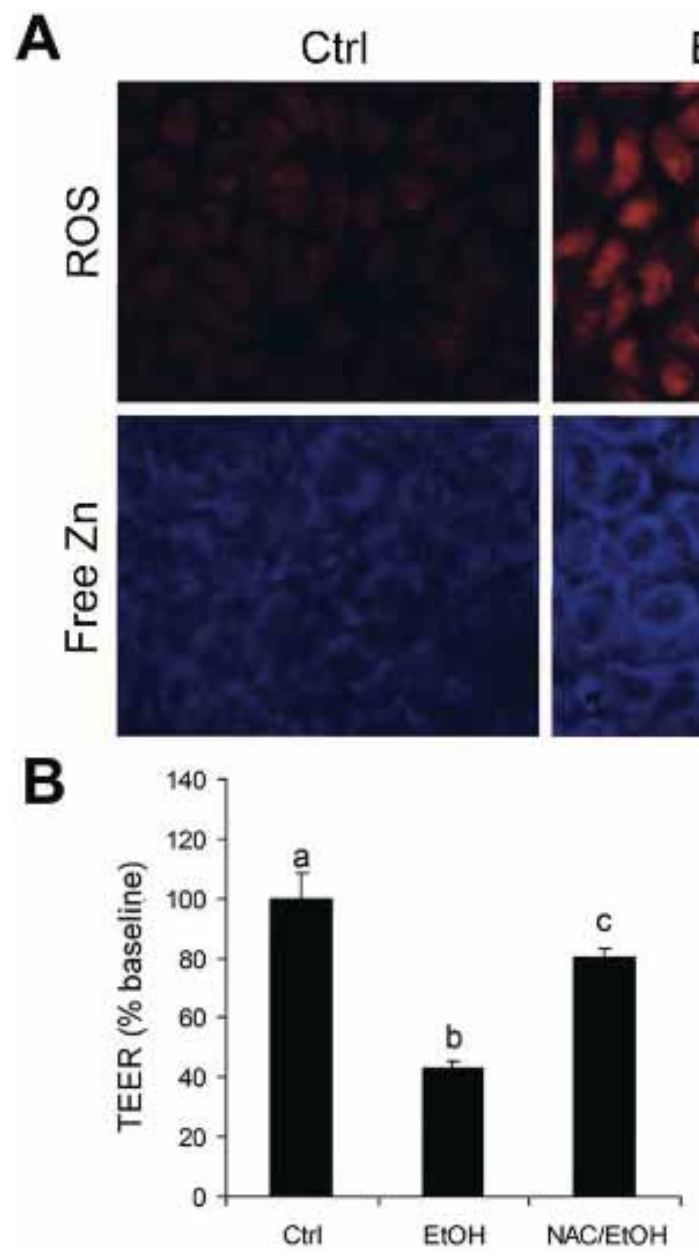

$\mathrm{EtOH}$
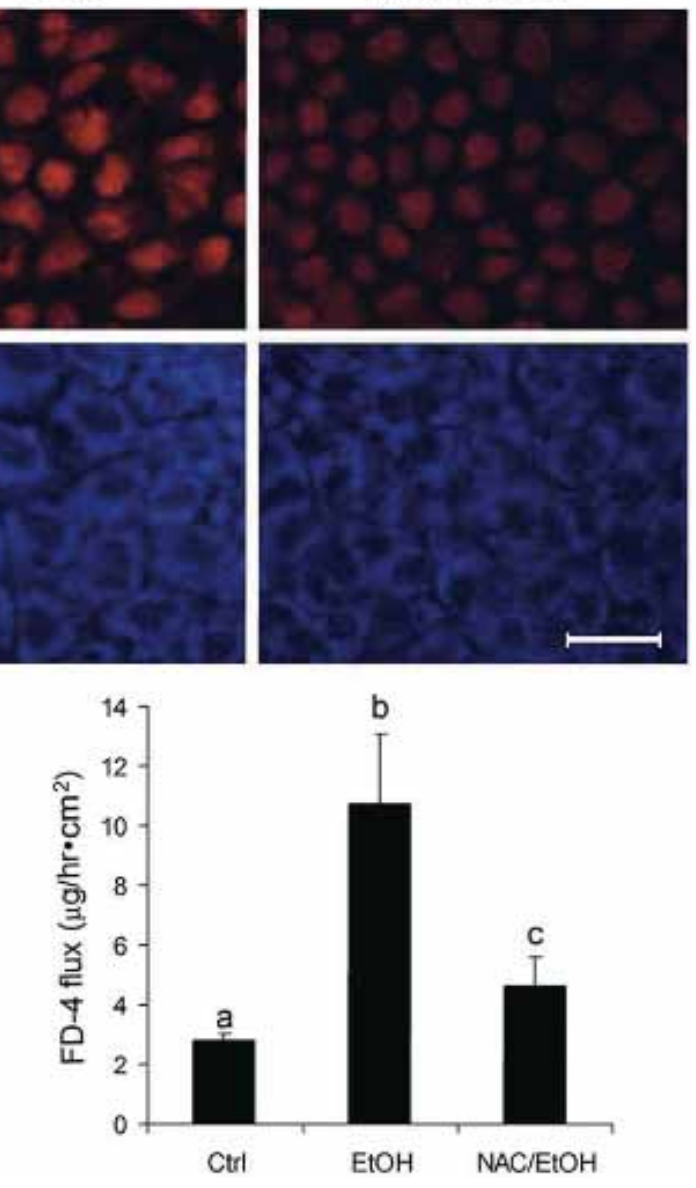

Fig. (3). Association of zinc release with ROS generation in alcohol-induced epithelial barrier disruption in Caco-2 cells. A. Fluorescence microscopy of ROS and free zinc. Caco- 2 cells were cultured on chamber slides and treated with $5 \%$ ethanol (v/v) for $5 \mathrm{hr}$ in the presence or absence of $2 \mathrm{mM}$ NAC. ROS and free zinc were detected by fluorescent microscopy after incubation with dihydroethidium (5 $\mu \mathrm{M})$ or Zinquin $(25 \mu \mathrm{m})$, respectively. Scale bar: $25 \mu \mathrm{m}$. B. Effect of NAC on alcohol-induced epithelial barrier disruption. Caco-2 cells were treated with 5\% ethanol with or without $2 \mathrm{mM}$ NAC pretreatment. Results are means \pm SD $(n=8)$. Significant differences $(p<0.05$, ANOVA) are identified by different letters. Ctrl: Control. EtOH: Ethanol. NAC: N-acetyl-cysteine. TEER: Trans-epithelial electronic resistance. 

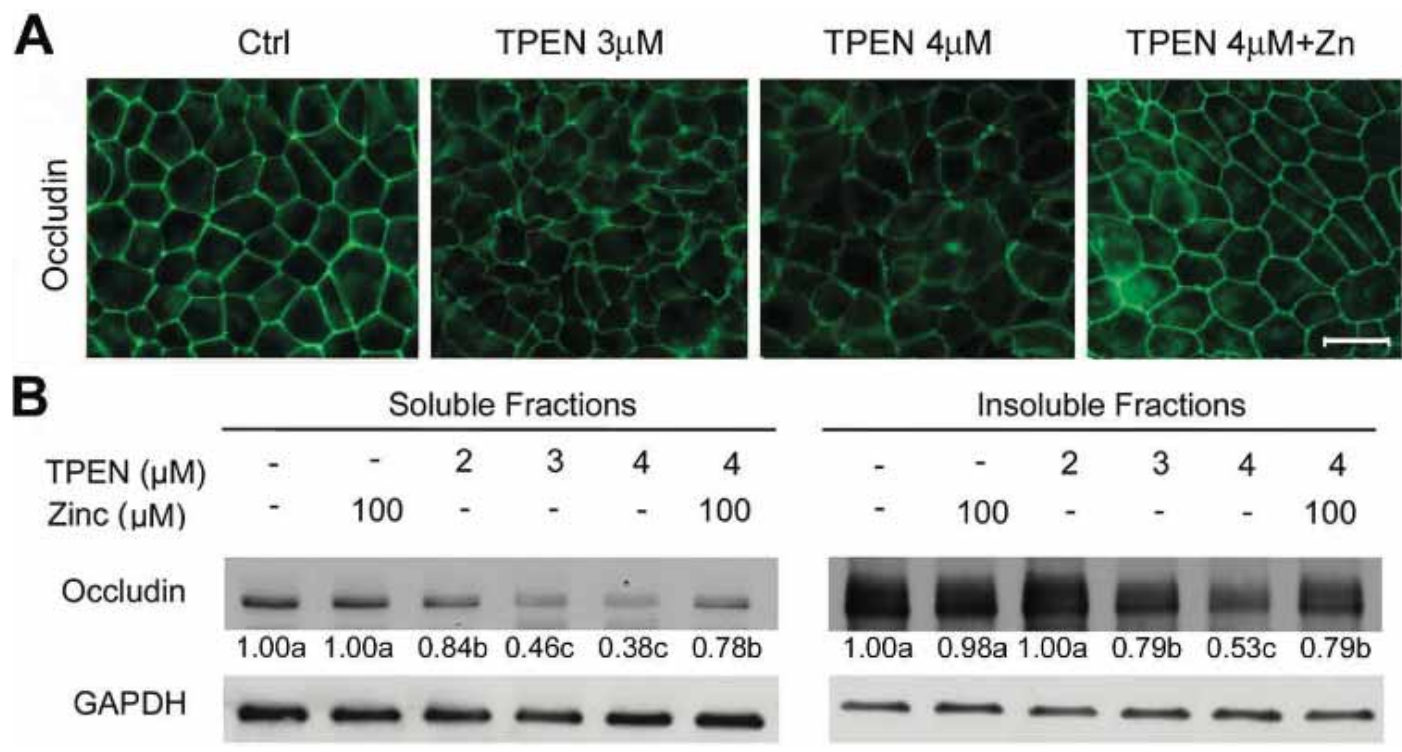

Fig. (4). Effect of zinc deprivation on the epithelial barrier of Caco-2 cells. Caco-2 cells were cultured on inserts and treated with TPEN at 2 , 3 and $4 \mu \mathrm{M}$ or $4 \mu \mathrm{M}$ TPEN plus $100 \mu \mathrm{M}$ zinc for $24 \mathrm{hr}$. A. Distribution of tight junction protein, occludin, was examined by immunofluorescence microscopy. Scale bar: $25 \mu \mathrm{m}$. B. Occludin protein level was determined by immunoblot analysis. Immunoblot bands were quantified by densitometry analysis and the ratio to GAPDH was calculated by setting the value of controls as one. Results are means \pm SD ( $n=4)$. Significant differences ( $p<0.05$, ANOVA) are identified by different letters. TPEN: N,N,N',N'-tetrakis(2-pyridylmethyl)ethylenediamine.

likely a major cause in alcohol-induced intestinal zinc deficiency. The relation between ROS and zinc deficiency in alcohol-induced intestinal barrier dysfunction could be that ROS reduce cellular zinc, and zinc deficiency, in turn, disassembles tight junction proteins.

Alcoholics commonly have a lower zinc intake [59]. To determine if zinc deficiency might interact with alcohol to induce epithelial barrier dysfunction, Caco-2 cells were cultured under minor zinc deficiency ( $2 \mu \mathrm{M}$ TPEN) condition, followed by treatment with alcohol [12]. Alcohol treatment reduced the distribution of claudin-1, occludin and ZO-1 at the tight junctions, while minor zinc deficiency alone did not affect the tight junction proteins. However, Caco-2 cells treated with alcohol and minor zinc deficiency showed a dramatic reduction of tight junction proteins including claudin-1, occludin and ZO-1 in both intercellular distribution and protein levels in comparison with alcohol alone [12]. In accordance, Caco-2 cells treated with zinc deficiency and alcohol showed significantly lower trans-epithelial electronic resistance and higher FITC-dextran permeability than alcohol alone [12]. These data suggest that minor dietary zinc deficiency may not directly affect the intestinal barrier but sensitize the deleterious actions of alcohol on the intestinal barrier.

\section{HNF-4 $\alpha$ INACTIVATION AND ALCOHOL-INDUCED DOWN-REGULATION OF TIGHT JUNCTION PROTEINS}

$\mathrm{HNF}-4 \alpha$, a zinc finger transcription factor, is mainly expressed in the liver, intestine, pancreas and kidney. HNF- $4 \alpha$ has been shown to be essential for enterocyte differentiation $[60,61]$. Although the role of HNF- $4 \alpha$ in the pathophysiology of intestinal diseases has not been well defined, a recent study showed that knockout of HNF- $4 \alpha$ in the intestinal epithelial cells in mice increased the susceptibility to experimental inflammatory bowl disease [60]. Importantly, the intestinal permeability was significantly higher in the intestinal epithelial HNF- $4 \alpha$ null mice in acute colitis. A recent study reported that $\mathrm{HNF}-4 \alpha$ inactivation is likely a novel molecular mechanism underlying alcohol-induced disassembly of tight junction proteins and dysfunction of the epithelial barrier [62].

HNF- $4 \alpha$ was detected in all the segments of the gastrointestinal tract except for the stomach [62], and its distribution gradually increased along the intestine from the proximal to the distal. In contrast, two other important intestinal zinc finger transcription factors, GATA-4 and SP1, were extensively distributed in the stomach, gradually decreased along the small intestine, and absent in the cecum and colon. Because chronic alcohol exposure caused zinc deficiency and gut leakiness in the distal intestine [12], HNF- $4 \alpha$ is potentially related in alcohol action. Indeed, chronic alcohol exposure significantly inhibited HNF-4 $\alpha$ DNA binding activity in all the intestinal segments, and the most prominent effect was in the ileum; a $70 \%$ decrease in DNA binding activity compared with $20-30 \%$ decreases in the duodenum, jejunum and colon (Fig. 5A). In accordance, chronic alcohol exposure significantly decreased HNF- $4 \alpha$ protein levels in all the intestinal segments with the greatest reduction in the ileum (Fig. 5B). Most importantly, a highly positive correlation was found between the intestinal HNF- $4 \alpha$ mRNA and the intestinal tight junction protein mRNAs, claudin-1 ( $\mathrm{r}=0.697)$, occludin ( $\mathrm{r}=0.547)$ and ZO-1 ( $\mathrm{r}=0.586)$ [62].

Caco-2 cell culture studies further defined the role of $\mathrm{HNF}-4 \alpha$ in regulation of tight junction proteins and epithelial barrier function [62]. Knockdown of HNF- $4 \alpha$ by siRNA transfection resulted in decreases in intercellular distribution (Fig. 6A) and protein levels (Fig. 6B) of occludin and other junction proteins in association with disruption of the epithelial barrier function. While previous studies demonstrated 

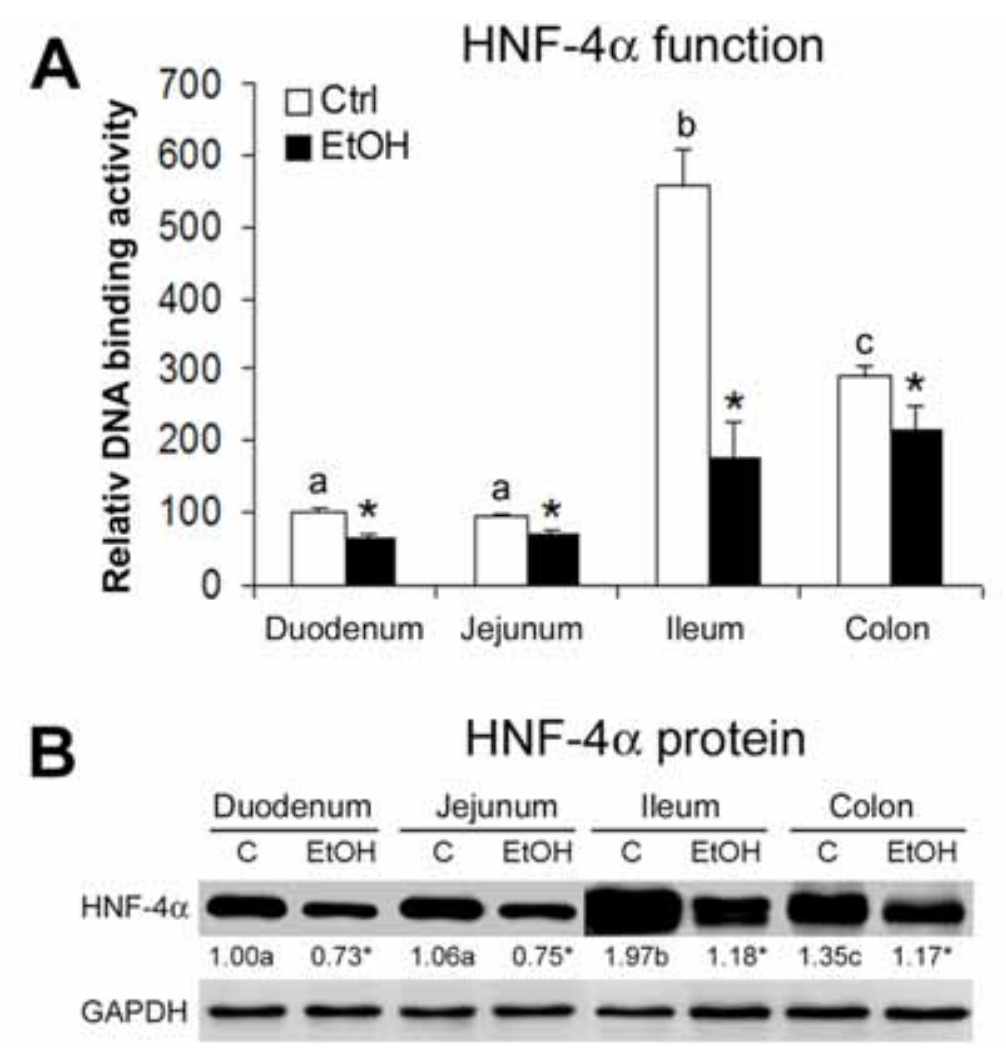

Fig. (5). Intestinal HNF-4 $\alpha$ in mice chronically fed alcohol for 4 weeks. A. HNF-4 $\alpha$ function was assessed by measuring DNA binding activity with a Trans-AM ${ }^{\mathrm{TM}}$ HNF Family Transcription Factor ELISA Kit. B. HNF-4 $\alpha$ protein levels were measured by immunoblot analysis. The immunoblot bands were quantified by densitometry analysis. Results are means \pm SD $(n=6$ in A; $n=3$ in B $)$. Significant differences $(p<0.05)$ among intestinal segments were determined by ANOVA and indicated by different letters. Significant differences $(p<0.05)$ between alcohol-fed and pair-fed mice for each intestinal segment were determined by $t$-test and indicated by *.

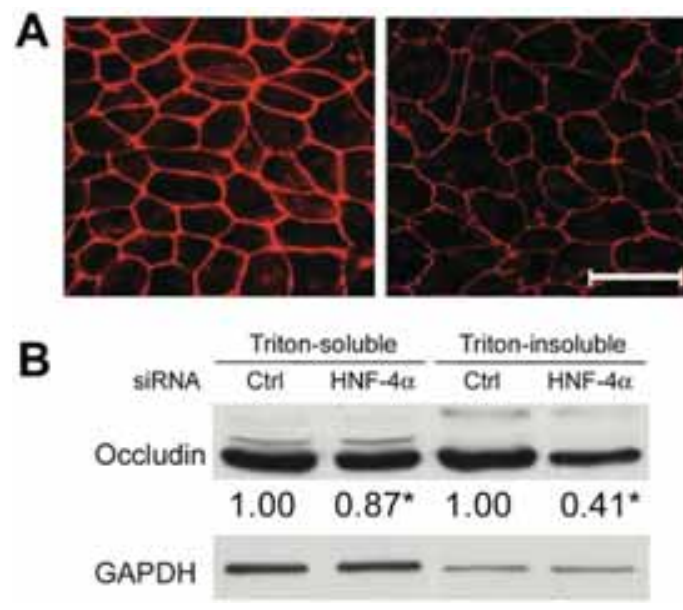

Fig. (6). Effects of HNF- $4 \alpha$ siRNA transfection on tight junction proteins. Caco-2 cells were transfected with HNF-4 $\alpha$ siRNA for 24 hr. A. Immunocytochemical staining of occludin. Scale bar: $25 \mu \mathrm{m}$. B. Immunoblot analysis of occludin protein level. The immunoblot bands were quantified by densitometry analysis. Results are means \pm SD $(n=3)$. Significant differences $(p<0.05)$ between control siRNA and HNF-4 $\alpha$ siRNA were detected by $t$-test and are indicated by $*$.

Ser/Thr phosphorylation- and microRNA (miRNA)dependent post-transcriptional mechanisms in alcoholinduced disassembly of tight junction proteins, HNF- $4 \alpha$ inactivation represents a transcriptional mechanism underlying alcohol effect on the epithelial barrier. Because alcoholinduced $\mathrm{HNF}-4 \alpha$ inactivation could be prevented by either NAC or zinc supplementation (Fig. 7A), HNF- $4 \alpha$ inactiva- tion is likely mediated by oxidative stress and zinc deficiency. Experimental zinc deprivation by TPEN suppressed HNF- $4 \alpha$ activity in a dose-dependent manner, but did not affect HNF-4 $\alpha$ protein level (Fig. 7B). Therefore, loss of zinc from the zinc finger of $\mathrm{HNF}-4 \alpha$ due to oxidative stress is likely a direct cause of $\mathrm{HNF}-4 \alpha$ inactivation. 

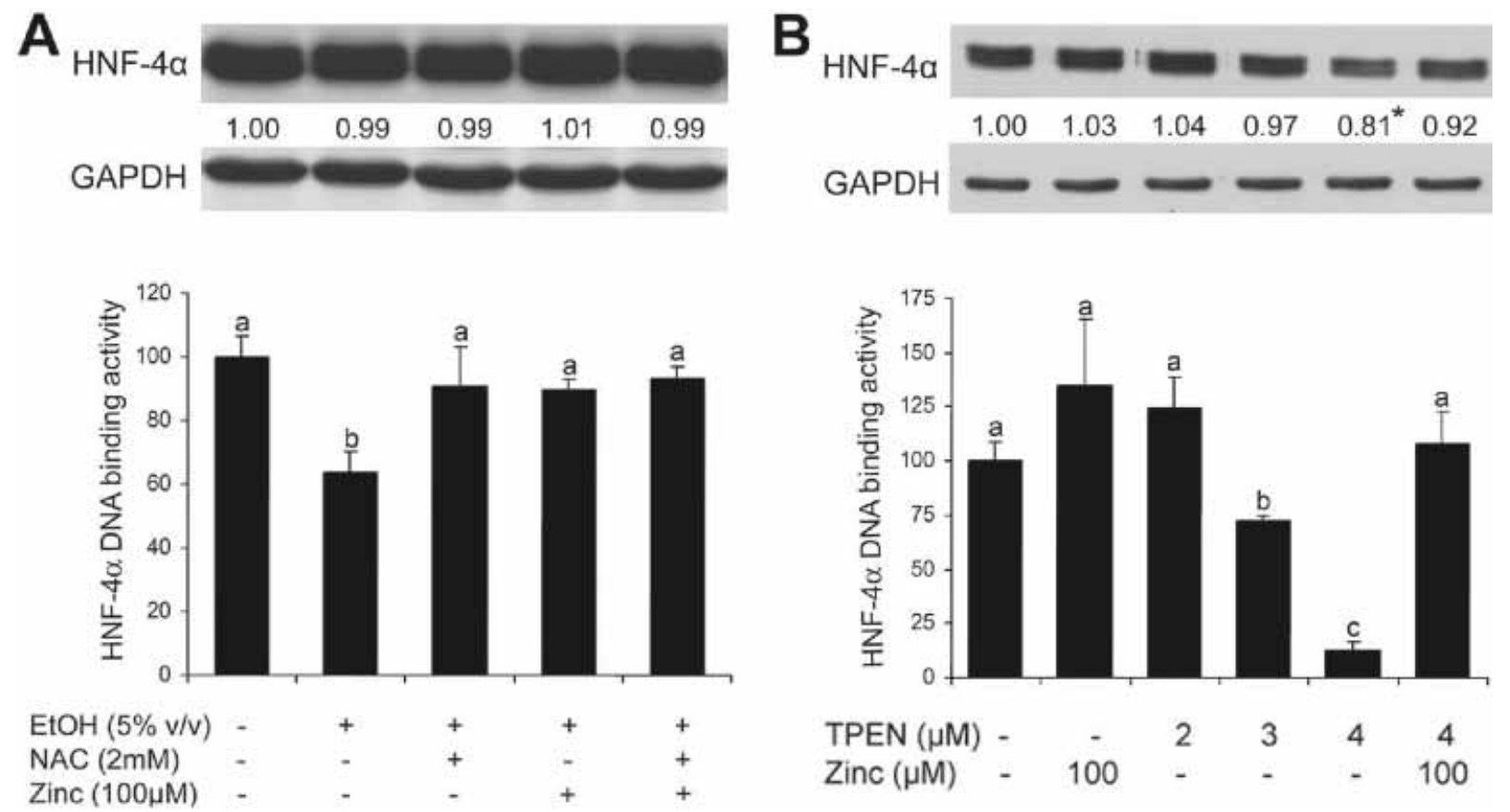

Fig. (7). Effects of alcohol and zinc deficiency on HNF-4 $\alpha$ protein and function in Caco-2 cells. For alcohol treatment, Caco-2 cells on Day 21 were exposed to $5 \%$ alcohol for $24 \mathrm{hr}$, and NAC and zinc were supplemented to determine the role of oxidative stress and zinc deprivation. For zinc deficiency study, Caco-2 cells on Day 21 were treated with TPEN at 2, 3 and $4 \mu \mathrm{M}$ or $4 \mu \mathrm{M}$ TPEN plus $100 \mu \mathrm{M}$ zinc for 24 hr. HNF- $4 \alpha$ protein levels were determined by immunoblot analysis. The immunoblot bands were quantified by densitometry analysis. HNF- $4 \alpha$ function was assessed by measuring HNF- $4 \alpha$ DNA binding activity with a Trans-AM ${ }^{\mathrm{TM}}$ HNF Family Transcription Factor ELISA kit. A. Effects of alcohol on HNF-4 $\alpha$. B. Effects of zinc deficiency on HNF-4 $\alpha$. Results are means \pm SD ( $n=6$ for DNA binding analysis; $n=3$ for immunoblot analysis). Significant differences $(p<0.05)$ were determined by ANOVA and are indicated by $*$ for immunoblot analysis and by different letters for DNA binding analysis.

\section{ZINC SUPPLEMENTATION IN CONTROL OF GUT PERMEABILITY}

Zinc supplementation has been shown to tighten the leaky gut under a variety of disease conditions. Patients with Crohn's disease showed an increase in gut permeability as indicated by a higher lactulose/mannitol ratio, which was attenuated by oral zinc sulfate supplementation for 8 weeks [63]. Oral zinc supplementation in patients with acute shigellosis or diarrhea improved the intestinal barrier function as indicated by reduction of lactulose:mannitol ratio [64, 65]. Evaluation of the tight junction ultrastucture by electron microscopy demonstrated that dietary zinc supplementation to rats with colitis reduced the number of opened tight junction complexes in the colon epithelium [66]. Zinc supplementation also prevented epithelial barrier dysfunction in association with indomethacin-induced of small intestine injury in a human study [67]. While zinc is known to stimulate proliferation and repair of the intestinal epithelium, these findings indicate that zinc also tightens the gut at subcellular level.

The effects of zinc pretreatment on alcohol-induced leaky gut were tested in a mouse model of acute alcohol intoxication [36]. Mice were treated with three doses of $\mathrm{ZnSO} 4$ at 5 $\mathrm{mg}$ elemental zinc/kg in a 12-hr interval prior to an oral dose of alcohol. Alcohol intoxication elevated plasma endotoxin level, which correlated well with liver pathological changes. Zinc pretreatment abrogated alcohol-induced endotoxemia and liver damage [36]. To determine the effect of alcohol intoxication and zinc supplementation on the gut permeabil- ity, endotoxin was administrated by gavage and the plasma level of endotoxin was measured. Alcohol treatment significantly increased the penetration of intragastrically administrated endotoxin to the blood, which as attenuated by zinc supplementation [36]. Therefore, zinc prevention of increased intestinal permeability is importantly involved in the inhibition of alcohol-induced liver damage in mice.

\section{CONCLUSION}

Disruption of the intestinal barrier is a causal factor in the development of alcoholic endotoxemia and hepatitis. Disassembly and/or reduction of proteins consisting of the apical junction complexes, particularly the tight junctions, are important mechanisms underlying the alcohol action on the intestinal barrier. Intestinal zinc deficiency correlates well with oxidative stress, decrease in tight junction proteins and increase in gut permeability. A mechanistic link could be that alcohol exposure via oxidative stress induces cellular zinc deficiency which, in turn, down-regulates tight junction proteins and causes epithelial barrier dysfunction. Zinc supplementation provides beneficial effects on gut permeability in a variety of disease conditions including ALD. Zinc critically modulates expression of tight junction proteins, thereby affecting the epithelial barrier. HNF- $4 \alpha$ is a zinc finger transcription factor and abundantly distributes in the distal intestine. Inactivation of HNF- $4 \alpha$ correlates well with alcoholinduced down-regulation of tight junction proteins in the intestine. Zinc release under oxidative stress accounts for alcohol-induced HNF- $4 \alpha$ inactivation. Therefore, zinc defi- 
ciency and HNF-4 $\alpha$ inactivation are likely a novel mechanism underlying alcohol effects on tight junction proteins at transcriptional level.

\section{CONFLICT OF INTEREST}

None declared.

\section{ACKNOWLEDGEMENTS}

None declared.

\section{REFERENCES}

[1] Powell SR. The antioxidant properties of zinc. J Nutr 2000; 130(5S Suppl): 1447S-54S.

[2] Giles NM, Watts AB, Giles GI, Fry FH, Littlechild JA, Jacob C. Metal and redox modulation of cysteine protein function. Chem Biol 2003; 10: 677-93.

[3] Kröncke KD, Klotz LO. Zinc fingers as biologic redox switches? Antioxid Redox Signal 2009; 11: 1015-27.

[4] Wilcox DE, Schenk AD, Feldman BM, Xu Y. Oxidation of zincbinding cysteine residues in transcription factor proteins. Antioxid Redox Signal 2001; 3: 549-64.

[5] Webster KA, Prentice H, Bishopric NH. Oxidation of zinc finger transcription factors: physiological consequences. Antioxid Redox Signal 2001; 3: 535-48.

[6] Roselli M, Finamore A, Garaguso I, Britti MS, Mengheri E. Zinc oxide protects cultured enterocytes from the damage induced by Escherichia coli. J Nutr 2003; 133: 4077-82.

[7] Jacquillet G, Barbier O, Cougnon M, et al. Zinc protects renal function during cadmium intoxication in the rat. Am J Physiol Renal Physiol 2006; 290: F127-37.

[8] Finamore A, Massimi M, Conti Devirgiliis L, Mengheri E. Zinc deficiency induces membrane barrier damage and increases neutrophil transmigration in Caco-2 cells. J Nutr 2008; 138: 1664-70.

[9] Joshi PC, Mehta A, Jabber WS, Fan X, Guidot DM. Zinc deficiency mediates alcohol-induced alveolar epithelial and macrophage dysfunction in rats. Am J Respir Cell Mol Biol 2009; 41: 207-16.

[10] Amasheh M, Andres S, Amasheh S, Fromm M, Schulzke JD. Barrier effects of nutritional factors. Ann NY Acad Sci 2009; 1165: 267-73.

[11] Zhang B, Guo Y. Supplemental zinc reduced intestinal permeability by enhancing occludin and zonula occludens protein-1 (ZO-1) expression in weaning piglets. Br J Nutr 2009; 102: 687-93.

[12] Zhong W, McClain CJ, Cave M, Kang YJ, Zhou Z. The role of zinc deficiency in alcohol-induced intestinal barrier dysfunction. Am J Physiol Gastrointest Liver Physiol 2010; 298: G625-33.

[13] Carr G, Wright JA, Simmons NL. Epithelial barrier resistance is increased by the divalent cation zinc in cultured MDCKII epithelial monolayers. J Membr Biol 2010; 237: 115-23.

[14] McGuckin MA, Eri R, Simms LA, Florin TH, Radford-Smith G. Intestinal barrier dysfunction in inflammatory bowel diseases. Inflamm Bowel Dis 2009; 15: 100-13.

[15] Rao R. Endotoxemia and gut barrier dysfunction in alcoholic liver disease. Hepatology 2009; 50: 638-44.

[16] Miele L, Valenza V, La Torre G, et al. Increased intestinal permeability and tight junction alterations in nonalcoholic fatty liver disease. Hepatology 2009; 49: 1877-87.

[17] Farhadi A, Gundlapalli S, Shaikh M, et al. Susceptibility to gut leakiness: a possible mechanism for endotoxaemia in non-alcoholic steatohepatitis. Liver Int 2008; 28: 1026-33.

[18] Semrad CE. Zinc and intestinal function. Curr Gastroenterol Rep 1999; 1: 398-403.

[19] Szabo G, Bala S. Alcoholic liver disease and the gut-liver axis. World J Gastroenterol 2010; 16: 1321-9.

[20] Rao RK, Seth A, Sheth P. Recent Advances in Alcoholic Liver Disease. I. Role of intestinal permeability and endotoxemia in alcoholic liver disease. Am J Physiol Gastrointest Liver Physiol 2004; 286: G881-4.

[21] Fujimoto M, Uemura M, Nakatani Y, et al. Plasma endotoxin and serum cytokine levels in patients with alcoholic hepatitis: relation to severity of liver disturbance. Alcohol Clin Exp Res 2000; 24:48S-54S.
[22] Hanck C, Rossol S, Bocker U, Tokus M, Singer MV. Presence of plasma endotoxin is correlated with tumour necrosis factor receptor levels and disease activity in alcoholic cirrhosis. Alcohol Alcohol 1998; 33: 606-8

[23] Urbaschek R, McCuskey RS, Rudi V, et al. Endotoxin, endotoxin neutralizing capacity, sCD14, sICAM-1, and cytokines in patients with various degrees of alcoholic liver disease. Alcohol Clin Exp Res 2001; 25: 261-268.http://ajpgi.physiology.org/cgi/external_ref? access_num $=000167131100015 \&$ link_type $=$ ISI

[24] Nanji AA, Khettry U, Sadrzadeh SM. Lactobacillus feeding reduces endotoxemia and severity of experimental alcoholic liver (disease). Proc Soc Exp Biol Med 1994; 205: 243-7.

[25] Adachi Y, Moore LE, Bradford BU, Gao W, Thurman RG. Antibiotics prevent liver injury in rats following long-term exposure to ethanol. Gastroenterology 1995; 108: 218-24.

[26] Zhou Z, Wang L, Song Z, Lambert JC, McClain CJ, Kang YJ. A critical involvement of oxidative stress in acute alcohol-induced hepatic TNF- $\alpha$ production. Am J Pathol 2003; 163: 1137-46.

[27] Bjarnason I, Peters TJ, Wise RJ. The leaky gut of alcoholism: possible route of entry for toxic compounds. Lancet 1984; 1 179-82.

[28] Keshavarzian A, Fields JZ, Vaeth J, Holmes EW. The differing effects of acute and chronic alcohol on gastric and intestinal permeability. Am J Gastroenterol 1994; 89: 2205-11.

[29] Keshavarzian A, Holmes EW, Patel M, Iber F, Fields JZ, Pethkar S. Leaky gut in alcoholic cirrhosis: a possible mechanism for alcohol-induced liver damage. Am J Gastroenterol 1999; 94: 200-7.

[30] Parlesak A, Schafer C, Schutz T, Bode JC, Bode C. Increased intestinal permeability to macromolecules and endotoxemia in patients with chronic alcohol abuse in different stages of alcohol-induced liver disease. J Hepatol 2000; 32: 742-7.http://ajpgi.physiology.org/cgi/ external_ref?access_num=10.1016/S0168-8278\%2800\%2980242-1 \&link_type=DOI

[31] Enomoto N, Takei Y, Hirose M, et al. Thalidomide prevents alcoholic liver injury in rats through suppression of Kupffer cell sensitization and TNF-alpha production. Gastroenterology 2002; 123: 291-300.

[32] Forsyth CB, Farhadi A, Jakate SM, Tang Y, Shaikh M, Keshavarzian A. Lactobacillus GG treatment ameliorates alcohol-induced intestinal oxidative stress, gut leakiness, and liver injury in a rat model of alcoholic steatohepatitis. Alcohol 2009; 43: 163-72.

[33] Mathurin P, Deng QG, Keshavarzian A, Choudhary S, Holmes EW, Tsukamoto H. Exacerbation of alcoholic liver injury by enteral endotoxin in rats. Hepatology 2000; 32: 1008-17.

[34] Keshavarzian A, Choudhary S, Holmes EW, et al. Preventing gut leakiness by oats supplementation ameliorates alcohol-induced liver damage in rats. J Pharmacol Exp Ther 2001; 299: 442-8.

[35] Keshavarzian A, Farhadi A, Forsyth CB, et al. Evidence that chronic alcohol exposure promotes intestinal oxidative stress, intestinal hyperpermeability and endotoxemia prior to development of alcoholic steatohepatitis in rats. J Hepatol 2009; 50: 538-47.

[36] Lambert JC, Zhou Z, Wang L, Song Z, McClain CJ, Kang YJ. Prevention of alterations in intestinal permeability is involved in zinc inhibition of acute ethanol-induced liver damage in mice. J Pharmacol Exp Ther 2003; 305: 880-6.

[37] Farhadi A, Banan A, Fields J, Keshavarzian A. Intestinal barrier: an interface between health and disease. J Gastroenterol Hepatol 2003; 18: 479-97

[38] Förster C. Tight junctions and the modulation of barrier function in disease. Histochem Cell Biol 2008; 130: 55-70.

[39] Kucharzik T, Walsh SV, Chen J, Parkos CA, Nusrat A. Neutrophil transmigration in inflammatory bowel disease is associated with differential expression of epithelial intercellular junction proteins Am J Pathol 2001; 159: 2001-9.

[40] Montalto M, Cuoco L, Ricci R, Maggiano N, Vecchio FM, Gasbarrini G. Immunohistochemical analysis of ZO-1 in the duodenal mucosa of patients with untreated and treated celiac disease. Digestion 2002; 65: 227-33.

[41] Basuroy S, Sheth P, Mansbach CM, Rao RK. Acetaldehyde disrupts tight junctions and adherens junctions in human colonic mucosa: protection by EGF and L-glutamine. Am J Physiol Gastrointest Liver Physiol 2005; 289: G367-G75.

[42] Ferrier L, Bérard F, Debrauwer L, et al. Impairment of the intestinal barrier by ethanol involves enteric microflora and mast cell activation in rodents. Am J Pathol 2006; 168: 1148-54. 
[43] Rao RK. Acetaldehyde-induced barrier disruption and paracellular permeability in Caco-2 cell monolayer. Methods Mol Biol 2008; 447: 171-83.

[44] Tang Y, Banan A, Forsyth CB, Fields JZ, Lau CK, Zhang LJ, Keshavarzian A. Effect of alcohol on miR-212 expression in intestinal epithelial cells and its potential role in alcoholic liver disease. Alcohol Clin Exp Res 2008; 32:355-64.

[45] Shimizu M, Lasker JM, Tsutsumi M, Lieber CS. Immunohistochemical localization of ethanol-inducible P450IIE1 in the rat alimentary tract. Gastroenterology 1990; 99: 1044-153.

[46] Hakkak R, Korourian S, Ronis MJ, Ingelman-Sundberg M, Badger TM. Effects of diet and ethanol on the expression and localization of cytochromes P450 2E1 and P450 2C7 in the colon of male rats. Biochem Pharmacol 1996; 51: 61-9.

[47] Bagchi D, Carryl OR, Tran MX, et al. Stress, diet and alcoholinduced oxidative gastrointestinal mucosal injury in rats and protection by bismuth subsalicylate. J Appl Toxicol 1998; 18: 3-13.

[48] Koyuturk M, Bolkent S, Ozdil S, Arbak S, Yanardag R. The protective effect of vitamin $\mathrm{C}$, vitamin $\mathrm{E}$ and selenium combination therapy on ethanol-induced duodenal mucosal injury. Hum Exp Toxicol 2004; 23: 391-98.

[49] Banan A, Choudhary S, Zhang Y, Fields JZ, Keshavarzian A. Ethanol-induced barrier dysfunction and its prevention by growth factors in human intestinal monolayers: evidence for oxidative and cytoskeletal mechanisms. J Pharmacol Exp Ther 1999; 291: 1075-85.

[50] Banan A, Fields JZ, Decker H, Zhang Y, Keshavarzian A. Nitric oxide and its metabolites mediate ethanol-induced microtubule disruption and intestinal barrier dysfunction. J Pharmacol Exp Ther 2000; 294: 997-1008.

[51] Banan A, Fields JZ, Zhang Y, Keshavarzian A. iNOS upregulation mediates oxidant-induced disruption of F-actin and barrier of intestinal monolayers. Am J Physiol Gastrointest Liver Physiol 2001; 280:G1234-46

[52] Rao RK, Baker RD, Baker SS, Gupta A, Holycross M. Oxidantinduced disruption of intestinal epithelial barrier function: role of protein tyrosine phosphorylation. Am J Physiol 1997; 273: G812-23.

[53] Banan A, Farhadi A, Fields JZ, Mutlu E, Zhang L, Keshavarzian A. Evidence that nuclear factor-kappa B activation is critical in oxidant-induced disruption of the microtubule cytoskeleton and barrier integrity and that its inactivation is essential in epidermal growth factor-mediated protection of the monolayers of intestinal epithelia. J Pharmacol Exp Ther 2003; 306: 13-28.

[54] Banan A, Zhang LJ, Farhadi A, Fields JZ, Shaikh M, Keshavarzian A. PKC-betal isoform activation is required for EGF-induced NFkappaB inactivation and IkappaB alpha stabilization and protection of F-actin assembly and barrier function in enterocyte monolayers. Am J Physiol Cell Physiol 2004; 286: C723-G38.

[55] Banan A, Zhang LJ, Shaikh M, Fields JZ, Farhadi A, Keshavarzian A. Inhibition of oxidant-induced nuclear factor-kappaB activation and inhibitory-kappaB alpha degradation and instability of F-actin cytoskeletal dynamics and barrier function by epidermal growth factor: key role of phospholipase-gamma isoform. J Pharmacol Exp Ther 2004; 309: 356-68.

[56] Kröncke KD. Cellular stress and intracellular zinc dyshomeostasis. Arch Biochem Biophys 2007; 463: 183-7.

[57] McClain CJ, Antonow DR, Cohen DA, Shedlofsky S. Zinc metabolism in alcoholic liver disease. Alcohol Clin Exp Res 1986; 10: 582-9.

[58] Ziegler TR, Evans ME, Fernández-Estívariz C, Jones DP. Trophic and cytoprotective nutrition for intestinal adaptation, mucosal repair, and barrier function. Annu Rev Nutr 2003; 23: 229-61.

[59] Watson RR. Drugs of Abuse and Immune Function. CRC Press $1990 ; 136$.

[60] Ahn SH, Shah YM, Inoue J, et al. Hepatocyte nuclear factor 4alpha in the intestinal epithelial cells protects against inflammatory bowel disease. Inflamm Bowel Dis 2008; 14: 908-20.

[61] Stegmann A, Hansen M, Wang Y, et al. Metabolome, transcriptome, and bioinformatic cis-element analyses point to HNF-4 as a central regulator of gene expression during enterocyte differentiation. Physiol Genomics 2006; 27: 141-55.

[62] Zhong W, Zhao Y, McClain CJ, Kang YJ, Zhou Z. Inactivation of hepatocyte nuclear factor-4\{alpha\} mediates alcohol-induced downregulation of intestinal tight junction proteins. Am J Physiol Gastrointest Liver Physiol 2010; 299: G643-51.

[63] Sturniolo GC, Di Leo V, Ferronato A, D'Odorico A, D'Inca R. Zinc supplementation tightens "leaky gut" in Crohn's disease. Inflamm Bowel Dis 2001; 7: 94-8.

[64] Roy SK, Behrens RH, Haider R, et al. Impact of zinc supplementation on intestinal permeability in Bangladeshi children with acute diarrhoea and persistent diarrhoea syndrome. J Pediatr Gastroenterol Nutr 1992; 15: 289-96.

[65] Alam AN, Sarker SA, Wahed MA, Khatun M, Rahaman MM. Enteric protein loss and intestinal permeability changes in children during acute shigellosis and after recovery: effect of zinc supplementation. Gut 1994; 35: 1707-11.

[66] Sturniolo GC, Fries W, Mazzon E, Di Leo V, Barollo M, D'inca R. Effect of zinc supplementation on intestinal permeability in experimental colitis. J Lab Clin Med 2002; 139: 311-15.

[67] Sivalingam N, Pichandi S, Chapla A, Dinakaran A, Jacob M. Zinc protects against indomethacin-induced damage in the rat small intestine. Eur J Pharmacol 2011; 654: 106-16. 